\title{
Les harmoniques de la pensée mystique de Jean de la Croix. Persistances et reprises poétiques: le P. Cyprien de la Nativité de la Vierge, Paul Valéry, Rainer Maria Rilke \\ The Harmony of the Mystical Thought of John of the Cross. Persistence and Poetical Resumption: P. Cyprien de la Nativité de la Vierge, Paul Valéry, Rainer Maria Rilke
}

\section{Dominique de Courcelles}

Paris Sciences Lettres Research University / Centre national de la recherche scientifique/ Ecole normale supérieure, Paris

FRANCE

dominique.decourcelles@club-internet.fr

[Hipogrifo, (issn: 2328-1308), 6.2, 2018, pp. 385-407]

Recibido: 10-01-2018 / Aceptado: 06-03-2018

DOI: http://dx.doi.org/10.13035/H.2018.06.02.31

Résumé. Les harmoniques de l'expérience spirituelle, mystique de Jean de la Croix au XVlème siècle en Espagne, se déploient en persistances et reprises poétiques infinies, toujours nouvelles, toujours recommencées. A partir de ses poèmes majeurs s'entrelacent de façon inattendue la traduction effectuée au XVIIème siècle par le carme P. Cyprien, la découverte de cette traduction par le poète Paul Valéry au début du XXème siècle, en parfaite conjonction avec sa propre recherche, ou encore leur résonance dans l'œuvre poétique de Rainer Maria Rilke, amoureux des «paysages » de l'Espagne, lecteur de Paul Valéry et de Jean de la Croix. Tout cela en attente d'autres entre-lacements et d'autres croisements. C'est toute une synes- 
thésie multiple, associée aux expériences sensibles, physiques ou spirituelles, de la vie, qui s'éprouve ainsi, pour se déployer en poésie pure par-delà les différences de langues, d'espaces et de temps.

Mots clés. P. Cyprien de la Nativité; Jean de la Croix; Mystique; Rainer Maria Rilke; Paul Valéry.

Abstract. The harmony of the spiritual, mystical experience of Jean de la Croix in the 16th century in Spain, unfolds itself in numerous poetic recreations, always new, always begun again. From his major poems interlace in an unexpected way the translation made in the 17th century by the Carmelite P. Cyprien, the discovery of this translation by Paul Valéry at the beginning of the 20th century, in perfect conjunction with its own research, or still their resonance in the poetic work of Rainer Maria Rilke, lover of the "landscapes" of Spain, and a reader of both Paul Valéry and Jean de la Croix... all this awaiting further relations and crossings. It is the whole multiple synaesthesia, associated with the sensitive, physical or spiritual experiences of life, that experiences itself in the pure poetry beyond the limits of languages, space and time.

Keywords. P. Cyprien de la Nativité; John of the Cross ; Misticism ; Rainer Maria Rilke; Paul Valéry.

Au XVlème siècle en Espagne, le carme Jean de la Croix (1542-1591), formé en lettres et en philosophie, mais également en sculpture et en peinture, choisit la poésie pour transmettre la «substance» de sa quête et de son expérience spirituelle. C'est dans ses trois poèmes que l'on désigne couramment comme ses trois poèmes majeurs, appelant leurs propres explications dans des commentaires en prose à la fois didactiques et conceptuels, que Jean a présenté ce qui a été dénommé par le poète Paul Valéry comme «les harmoniques de sa pensée mystique», si I'on entend par harmoniques les assonances poétiques qui communiquent des signifiances ${ }^{7}$. Ce dont Jean lui-même rend compte en ces termes dans son prologue au Cántico espiritual, ces canciones que tratan del ejercicio de amor entre el alma y el esposo Cristo:

Por haberse estas canciones compuesto en amor de abundante inteligencia mística, no se podrán declarar al justo, ni mi intento será tal, sino sólo dar alguna luz en general... la sabiduría mística... no ha menester distintamente entenderse para hacer efecto de amor y afición en el alma... por tanto seré bien breve?

Et il ajoute: «Las verdades divinas... por amor... no solamente se saben, mas juntamente se gustan» ${ }^{3}$. Ce gustar - savourer sensiblement- est nécessairement associé à tout un ensemble d'expériences sensibles. Jean écrit dans son commentaire de la canción 15 du Cántico espiritual cette réflexion extrêmement significative:

1. Morier, Dictionnaire de poétique et de rhétorique, art. «Harmoniques», pp. 499-500.

2. San Juan de la Cruz, Cántico espiritual y poesía completa, p. 4.

3. San Juan de la Cruz, Cántico espiritual y poesía completa, p. 5. 
Porque acaecerá que vea el alma en sí las flores de «las montañas» que arriba dijimos, que son la abundancia y grandeza y hermosura de Dios; y en éstas entretejidos los lirios de «los valles nemorosos», que son descanso, refrigerio y amparo; y luego allí entrepuestas las rosas olorosas de las «ínsulas extrañas», que decíamos ser las extrañas noticias de Dios; y también embestirla el olor de las azucenas de «los ríos sonorosos», que decíamos era la grandeza de Dios, que hinche toda el alma; y entretejido allí y enlazado el delicado olor del jazmín del «silbo de los aires amorosos», de que también dijimos gozaba el alma en este estado; y, ni más ni menos, todas las otras virtudes y dones que decíamos del conocimiento sosegado, y «la callada música», y «soledad sonora» $y$ «la sabrosa y amorosa cena». Y es de tal manera el gozar y sentir estas flores juntas... ${ }^{4}$

Si le savourer par les sens a bien à voir avec la jouissance qui accompagne l'expérience spirituelle de l'union mystique et donc avec les sens spirituels - présenté comme ce conocimiento sosegado, la connaissance tranquille, c'est toute une synesthésie multiple qui est ici impliquée, charnelle ou physique et spirituelle. En écho à la perception visuelle dérivée de l'image des fleurs, les sensations de l'odorat -el olor-s'entrecroisent et s'entrelacent en procédant du silbo - son- et des aires -toucher, contact. Si Dieu exerce sur les puissances des sens un impact simultané, les flores juntas expriment bien cette fusion sensorielle, physique et spirituelle, cette synesthésie cumulative et multiple. C'est ainsi que les nuances du sens n'ont pas forcément d'importance, parce que le possible de la poésie, établie dès l'origine dans l'ineffable, est d'une richesse obscure et inépuisable. «Ce qui se produit avec la poésie, c'est quelque chose d'absolu» ${ }^{5}$, écrit la philosophe Maria Zambrano, grande lectrice des poésies de Jean de la Croix. L'important est de revenir aux flores juntas, de se retrouver avec le monde qui est l'abondance, la grandeur et la beauté de Dieu ineffable: «un sens au-delà de tout sens, la raison ultime au-dessus de toute raison» ${ }^{6}$. Jean de la Croix ne manque pas d'affirmer: «El lenguaje de Dios... excede todo sentido ${ }^{7}$... el lenguaje de Dios al alma va de puro espíritu [a] espíritu puro $^{8} \ldots$... Et il ajoute:

... cuán bajos y cortos y en alguna manera impropios son todos los términos y vocablos con que en esta vida se trata de las cosas divinas, y cómo es imposible por vía y modo natural, aunque más alta y sabiamente se hable en ellas, poder conocer ni sentir de ellas como ellas son, sin la iluminación de esta mística teología... secreta9 ${ }^{9}$.

Cet effort de «communication de l'incommunicable», pour reprendre l'expression de Theodor W. Adorno, ce secret ou cette obscurité, est explicitement indiqué dans les prologues de Jean aux commentaires de ses poèmes, en particulier dans le prologue du Cántico espiritual:

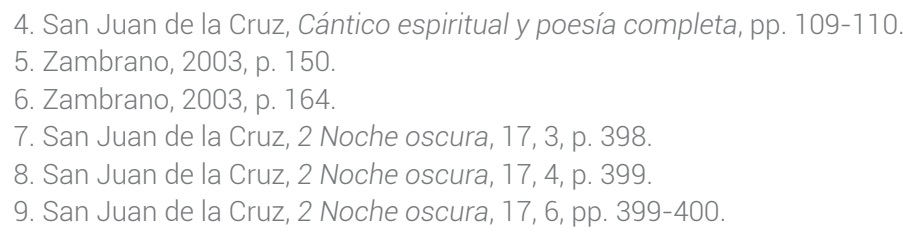


Sería ignorancia pensar que los dichos de amor en inteligencia mística (cuales son de las presentes canciones) con alguna manera de palabras se puedan bien explicar... ¿Quién podrá manifestar con palabras lo que hace sentir a las almas amorosas?... Cierto nadie lo puede... Que ésta es la causa por que con figuras, comparaciones y semejanzas antes rebosan algo de lo que sienten, y de la abundancia del espíritu vierten secretos misterios... ${ }^{10}$.

Ces paroles visent en effet à faire entendre les «mystères secrets», silencieux, d'où elles jaillissent et où elles conduisent. Ce silence n'est pas suspension de la parole et des mots, mais suspension du sens, mystère. D'où l'intensité, esa misma abundancia e impetu -l'abondance et l'impétuositél1 -, de cette voix muette qui porte en elle l'autre, mystérieux et inconnu, el que habla -celui qui parle ${ }^{12}$. L'oralité ressortit alors intimement à la démarche mystique.

C'est ainsi qu'il se produit dans les deux premières strophes de la Noche oscura un phénomène d'assonance à la fois imperceptible et sensible, une impressionnante fréquence de la voyelle $a$, insistant aussi bien à la rime que dans le corps du texte:

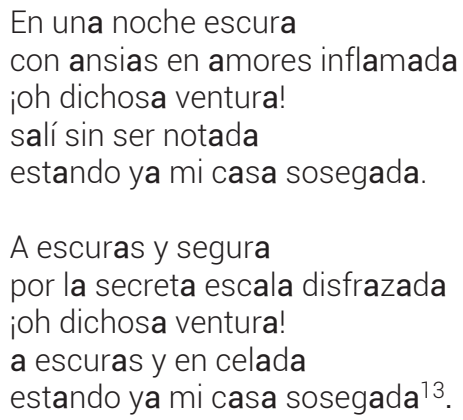

Ce tramé vocalique en a fait entendre quelque chose qui n'est ni sens ni musique, mais de l'ordre de la scansion, du rythme, du mouvement. Cette réussite de la sortie secrète de la casa sosegada en una noche oscura est aussi celle dans laquelle l'euphonie atteint un haut degré d'élaboration.

Précisément, dans son commentaire de la Noche oscura, Jean de la Croix pour caractériser le dire de l'indicible prend l'exemple du prophète Jérémie qui, après avoir parlé avec Dieu, se trouva incapable de «manifester» et de «dire extérieurement» et «ne sut que dire sinon A, A, A»: «La cortedad del manifestarlo y hablarlo exteriormente mostró Jeremías cuando, habiendo Dios hablado con él, no supo qué decir sino A, A, A $(1,6)$ » $^{14}$. Et dans le Dialogue de l'âme de Catherine de Sienne, on retrouve cette même assonance de balbutiement. A la fin de la huitième partie du Dialogue, l'âme «comme ivre» de Dieu perd les mots:

10. San Juan de la Cruz, Cántico espiritual y poesía completa, pp. 3-4.

11. San Juan de la Cruz, Cántico espiritual y poesía completa, p. 3.

12. San Juan de la Cruz, Subida del Monte Carmelo, p. 89.

13. San Juan de la Cruz, Cántico espiritual y poesía completa, p. 205.

14. San Juan de la Cruz, 2 Noche oscura, 2, 17, 4, p. 398. 
Et que dirai-je? Je ferai comme le bègue et dirai: $A$, a; parce que je ne sais rien dire d'autre, parce que la langue finie ne peut exprimer le sentiment de l'âme qui infiniment te désire $(153)^{15}$.

Ce balbutiement qui n'en finit pas de se répéter, exprimant l'impossibilité d'exprimer l'ineffable, Jean de la Croix l'a évoqué avec une autre assonance dans le Cántico espiritual: «Un no sé qué que quedan balbuciendo» ${ }^{16}$. Et l'on ne doit pas oublier ici que c'est par cette même forme insistante et répétée d'assonance, qui est beaucoup plus qu'une simple pratique allitérative, que saint Augustin, bien des siècles auparavant, entendant une comptine d'enfant dans le jardin de Milan saisit le livre par lequel il va achever son processus de conversion. «Tolle-lege-tolle-lege» ${ }^{17}$.

La sabiduría mística, la cual es por amor, de que las presentes canciones tratan - dit encore Jean de la Croix dans le prologue du Cántico espiritual- no ha menester distintamente entenderse para hacer efecto de amor y afición en el alma, porque es a modo de la fe, en la cual amamos a Dios sin entenderle ${ }^{18}$.

Car la parole poétique qui ici excède les sens, comme un balbutiement ou une comptine d'enfant, libère de la pesanteur des choses du monde et repousse la naturelle et élémentaire obscurité pour laisser place à la lumière unifiante et paradoxalement secrète du divin. Elle est un instrument de connaissance qui traduit le parcours mystique du poète par des symboles verbaux, assonances ou images concrètes. Selon la tradition, le début du Cántico espiritual aurait été inspiré à Jean de la Croix par une refrain d'amour entendu depuis la prison de Tolède où il fut enfermé de décembre 1577 jusqu'à son évasion à la mi-août 1578.

Jean de la Croix procède par images et analogies - figuras, comparaciones y semejanzas-, il rapproche des réalités séparées et son art poétique est un art du caché, de l'ailleurs, de l'invisible, où le symbole occupe une grande place. De la nuit obscure à la flamme éblouissante son langage poétique est un médiateur entre le réel et le mystère divin. Le rôle du lecteur, ici les moniales carmélites, filles de Thérèse de Jésus, est essentiel: elles doivent savoir interpréter les images «mystérieuses» qui leur sont proposées, vérités et «mystères secrets». Jean de la Croix n'éprouve aucune inquiétude à ce sujet. II écrit à la Mère Anne de Jésus, si aimée par Thérèse de Jésus: «Aunque a Vuestra Reverencia le falte el ejercicio de teología escolástica, ... no le falta el de la mística...»19.

Malgré ou à cause de leur tradition à la fois savante, le lyrisme italianisant de Garcilaso de la Vega (1501-1536), premier grand poète de la Renaissance espagnole, et populaire - les chansons, romances, etc. les poèmes de Jean de la Croix relèvent aussi de la tradition hébraïque du Cantique des Cantiques et de la tradition

\footnotetext{
15. Je me permets de renvoyer ici à mon analyse dans Le «Dialogue» de Catherine de Sienne, p. 76. 16. San Juan de la Cruz, Cántico espiritual y poesía completa, p. 13.

17. Je me permets de renvoyer à mon analyse dans Augustin génie de l'Europe, 1998, pp. 52-54; Agustín o el genio de Europa, 1998, pp. 53-54.

18. San Juan de la Cruz, Cántico espiritual y poesía completa, p. 4

19. San Juan de la Cruz, Cántico espiritual y poesía completa, p. 5.
} 
arabe qui privilégie les beautés de détail et inspire les images d'une symbolique très proche de celle du soufisme ${ }^{20}$. Est-ce pour cela que Jean de la Croix, ainsi que son œuvre, va être victime d'incompréhension, méfiance et ostracisme de la part de ses propres frères? II meurt en 1591, son œuvre n'est éditée en Espagne qu'en 1618, à Alcalá, dans une édition partielle: le commentaire au Cántico espiritual n'y figure pas. La première édition espagnole n'est publiée qu'en 1630. Jean de la Croix n'est béatifié qu'en 1675 et canonisé en 1726.

C'est vers 1910 que le poète Paul Valéry (1871-1945), grand admirateur de Stéphane Mallarmé dont il fréquente les rencontres littéraires, découvre «par hasard» dans les poèmes de Jean de la Croix «l'un des plus parfaits poètes de France dans le R. P. Cyprien de la Nativité de la Vierge, carme déchaussé jusqu'ici à peu près inconnu», grâce à l'ouvrage intitulé Les Fuvres Spirituelles du B. Père Jean de la Croix, premier carme déchaussé de la Réforme de N.D. du Mont Carmel, et coadjuteur de la Saincte Mère Térèse de Jésus, etc. etc. Le tout traduit en français par le R.P. Cyprien de la Nativité de la Vierge, Carme déchaussé, $1647^{21}$.

Immédiatement Paul Valéry explique: «Je ne suis pas grand lecteur d'ouvrages mystiques... La compréhension y est, sans doute, nécessaire: elle est fort loin d'être suffisante» ${ }^{22}$. Dans les trois poèmes traduits de Jean de la Croix, Paul Valéry reconnait une «pensée pure qui a sa poésie». Ecoutons ici Paul Valéry:

II n'est pas interdit de penser que le mode adopté par Jean de la Croix pour communiquer ce qu'on peut nommer les harmoniques de sa pensée mystique, tandis que cette pensée elle-même s'exprime à découvert dans le voisinage immédiat, pourrait être employé au service de toute pensée abstraite ou approfondie, de celles qui peuvent cependant exciter une émotion. Il est de telles pensées, et il existe une sensibilité des choses intellectuelles: la pensée pure a sa poésie ${ }^{23}$.

La «théologie mystique secrète» de Jean de la Croix serait donc exprimée en «poésie pure», pour reprendre la belle formule de Paul Valéry, à la suite de Stéphane Mallarmé. Quand Mallarmé parle du langage essentiel, il l'oppose à la fois au langage ordinaire et au langage de la pensée; c'est dans la parole poétique que le langage parle comme essentiel et que le poète fait œuvre de pur langage. Maria Zambrano explique:

Baudelaire, Valéry ont réalisé et, en même temps, défıni la «poésie pure». La poésie pure est une affirmation, une croyance en la poésie, en sa substantialité, en sa solitude, en son indépendance. La poésie pure... a fondé l'idée que la poésie est tout. Tout, entendons-nous, par rapport à la métaphysique; tout, quant à la connaissance, tout quant à la réalisation essentielle de l'homme. II suffit au poète de faire de la poésie pour exister; c'est la forme la plus pure de réalisation de l'essence humaine... il y a désormais une éthique du poète dans l'acte poétique. Une éthique

20. López-Baralt, prologue, San Juan de la Cruz, Obra completa, 1991.

21. Jean de la Croix, Les Cantiques spirituels, p. 8.

22. Jean de la Croix, Les Cantiques spirituels, p. 8.

23. Jean de la Croix, Les Cantiques spirituels, p. 14. 
qui consiste à être éveillé, précisément, qui consiste en cette veille obstinée, ce sacrifice constant...ce que dit Paul Valéry... ${ }^{24}$

C'est en «voyant», «lisant» et «murmurant» la traduction de la première strophe de la Noche oscura que Valéry acquiert ce qu'il dénomme la «certitude de poésie»:

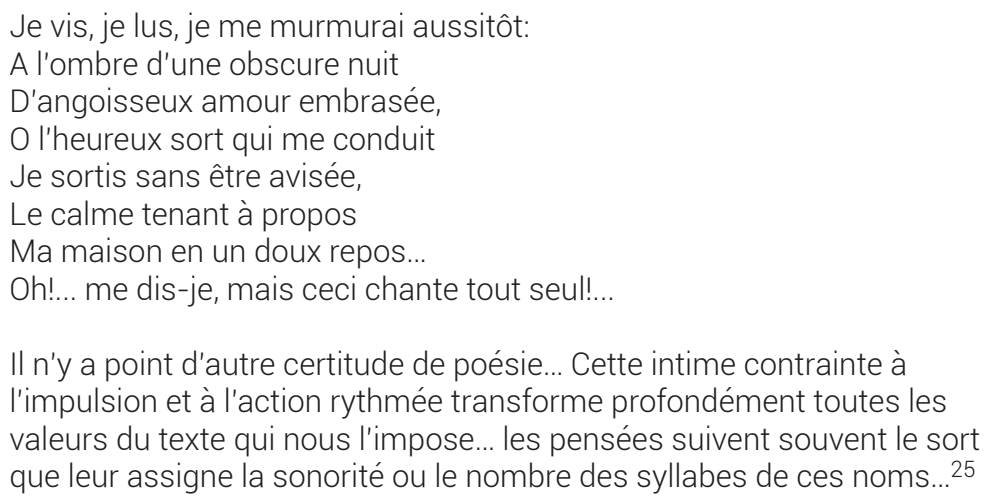

On retrouve ici la transposition sensorielle chère à Jean de la Croix, sa perception simultanée qui est le ressort de son expérience spirituelle, cette synesthésie multiple.

«Faire vivre quelque différente vie... dans un tout autre mode et comme dans un tout autre temps» ${ }^{26}$, dit Paul Valéry qui a lui-même pratiqué cet art de la correspondance et des harmoniques, par exemple dans ce passage très significatif du Cantique des Colonnes évoquant le mouvement et la quête:

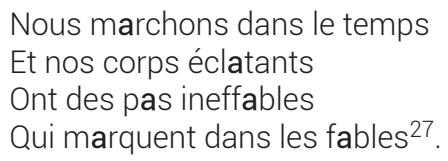

La fréquence de la voyelle a (renforcée dans les deux premiers vers du poème de Paul Valéry par la syllabe tem/tan et dans les deux derniers par la syllabe fabl-) est bien propre à marquer le mouvement, l'avancée, comme dans la langue castillane des deux premières strophes de la Noche oscura de Jean de la Croix. Paul Valéry explique, dans la perspective de Mallarmé: «Mon inspiration n'est pas verbale. Elle ne procède pas par mots. Plutôt par formes musicales» ${ }^{28}$.

Car les nuances du sens ne sont pas aussi importantes que la sonorité. Comme l'exprime encore le poète à propos du texte du P. Cyprien traduisant Jean de la Croix:

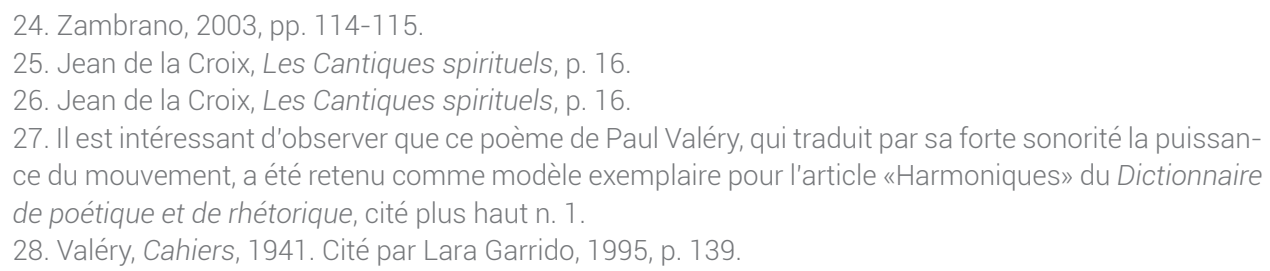


«Les pensées suivent souvent le sort que leur assigne la sonorité ou le nombre des syllabes de ces noms; elles s'enrichissent des similitudes et des contrastes qu'elles éveillent ${ }^{29}$.

Ayant lu et relu les vers en français de Jean de la Croix, s'étant ensuite attaché à «regarder à l'espagnol», Paul Valéry admire sans réserve la «fidélité absolue» de la traduction, affirmant en connaisseur qui n'aime que «ce qui donne le sentiment de la perfection» que

Ce faisant le P. Cyprien a enrichi notre poésie, quoique de la manière la plus discrète... d'un très mince recueil, mais de la plus certaine et de la plus pure qualité30.

Est-il dans La Fontaine même, ou dans Verlaine, chant plus fluide, fluide, mais non lâche, évadé plus heureusement du silence? ${ }^{31}$

«Faire des vers à l'état pur», explique ensuite Paul Valéry, a consisté pour le P. Cyprien à ne laisser dans son œuvre que «la façon de la forme», cependant que les idées, les images, le choix des mots -figuras, comparaciones y semejanzas-appartenaient à Jean de la Croix. C'est ainsi que le P. Cyprien (1605-1680), «personne singulièrement imperceptible...» que... Henri Bremond... semble avoir ignorée », qui «s'appelait dans le siècle André de Compans... et eut d'abord une charge de finances» ${ }^{32}$, qui n'a pas craint de traduire un fondateur qui n'est ni béatifié ni canonisé, qui est l'auteur d'une importante œuvre littéraire, a atteint la perfection poétique. Telle est la fidélité de la traduction.

Ce que je nomme Perfection élimine la personne de l'auteur; et par là n'est pas sans éveiller quelque résonnance mystique, -comme le fait toute recherche dont on place délibérément le terme «à l'infini». Rien de moins moderne, car il ne s'agit guère plus aujourd'hui que de se faire connaître... ${ }^{33}$

Avec Paul Valéry on peut estimer que le P. Cyprien, en traduisant les œuvres poétiques de son maître Jean de la Croix, s'est parfaitement lancé à sa suite dans l'aventure de chercher et de dire l'indicible, d'exprimer l'ineffable, cet ineffable que la philosophe Maria Zambrano a défini ainsi en deux sens:

ce qui est ineffable d'être proche, d'être charnel; ce qui est ineffable aussi d'être inaccessible, d'être un sens au-delà de tout sens, la raison ultime au-dessus de toute raison. Tel est le drame qu'a humblement vécu tout poète ${ }^{34}$.

Maria Zambrano considère que, si la question de la poésie pure a été posée avec Mallarmé, elle s'est compliquée avec Valéry. Avec Mallarmé la poésie a tenté de se

29. Jean de la Croix, Les Cantiques spirituels, p. 17.

30. Jean de la Croix, Les Cantiques spirituels, p. 18.

31. Jean de la Croix, Les Cantiques spirituels, p. 19.

32. Jean de la Croix, Les Cantiques spirituels, pp. 22-24.

33. Jean de la Croix, Les Cantiques spirituels, p. 22.

34. Zambrano, 2003, p.164. 
définir par voie purement négative. Mallarmé parle d'«absences», car les choses sont, dans la poésie, par leur absence même, soumises au cours du temps, c'està-dire par ce qu'elles ont de plus vrai, leur pure essence. Pour Valéry, c'est la poésie même qui est essence pure; le poétique, cette alliance intime du son et du sens, mystérieux, mystique, «puissance sonore immédiate», ne s'obtient et ne se saisit que par approximation. Pensée et poésie sont liées.

Une œuvre, écrit-il, et ce terme s'applique aussi bien aux «EFuvres spirituelles» originales de Jean de la Croix, selon le titre donné par le P. Cyprien à ses traductions, qu'à ces traductions en tant que telles, «doit inspirer le désir de la reprendre, de s'en redire les vers, de les porter en soi pour un usage intérieur indéfini»35.

«Danser en étant chargé de chaînes», pour reprendre encore une expression de Paul Valéry, c'est ce qu'ont su faire les poètes qui ont souhaité développer les harmoniques de la pensée mystique de Jean de la Croix et, en particulier, le «singulier» traducteur P. Cyprien au XVIlème siècle, dont Paul Valéry prend soin de citer les propos:

Je rendray cet hommage à la vérité, qui est que le travail dont vous jouyssez à présent en l'estat que vous avez la version de ces œuvres, est une chose cachée, et qui ne peut jamais estre congneuë que de ceux qui prendront la peine de confronter l'original entier avec le Françoys... principalement le Cantique Spirituel... pourroit bien passer pour une œuvre nouvelle... ${ }^{36}$

Admirant sans réserve le traducteur poète du XVIIème siècle et son «œuvre nouvelle » de poésie pure associée à tout un ensemble d'expériences sensibles - «ceci chante tout seul»-, et souhaitant s'en inspirer dans sa propre poésie, Paul Valéry a lui-même été traduit avec la plus grande admiration et bonheur par son célèbre contemporain, le poète Rainer Maria Rilke. Ainsi trouvent de nouvelles persistances et reprises les harmoniques si singulières de la pensée mystique de Jean de la Croix: «La pensée pure a sa poésie».

Lorsqu'il découvre Paul Valéry, en ce début de XXème siècle ravagé par la première Guerre mondiale, Rainer Maria Rilke (1875-1926), qui a longuement lu et médité la vie des saints, reflète déjà dans son œuvre poétique l'importance de la nuit obscure, la vision de sa propre misère, l'aspiration à l'élévation lumineuse et la quête de la transcendance, le désir de l'union totale avec l'infini, toutes ces étapes spirituelles si bien décrites par Jean de la Croix. Mais il est également amoureux de la langue et des mots, de la couleur et de l'éclat des sonorités et de leurs assonances dont la langue allemande est si riche. Rilke, qui se sentira proche de la France après ses voyages à Paris et surtout lors de son installation en Valais de langue française, ne s'est jamais lassé de dire l'importance qu'avait eue pour lui la découverte de la poésie de Paul Valéry. II lui voue une admiration sans réserve, il est frappé par la rigueur de sa conception poétique -«la poésie pure»- et par sa sensualité raffinée. Et il deviendra un traducteur inégalé de ce poète. 
«Un jour, j'étais seul, j'attendais, toute mon œuvre attendait. Un jour j'ai lu Valéry, j'ai su que mon attente était finie», écrit Rilke à l'écrivain Monique Saint-Hélier en 1923.

Car Rilke, comme Valéry, veut, dès ses premiers poèmes, inviter à la pureté de l'espace et à la pure poésie, rejoignant ainsi sans le savoir, tout en le sachant beaucoup plus tard, le mystique Jean de la Croix découvert par Paul Valéry à travers la traduction du P. carme Cyprien de la Nativité de la Vierge.

Dans l'une de ses premières œuvres, le Livre de la vie monastique, première partie du Livre d'Heures - Stundenbuch composé en mai 1900 au cours de son deuxième voyage en Russie, Rilke s'adresse à Dieu en ces termes:

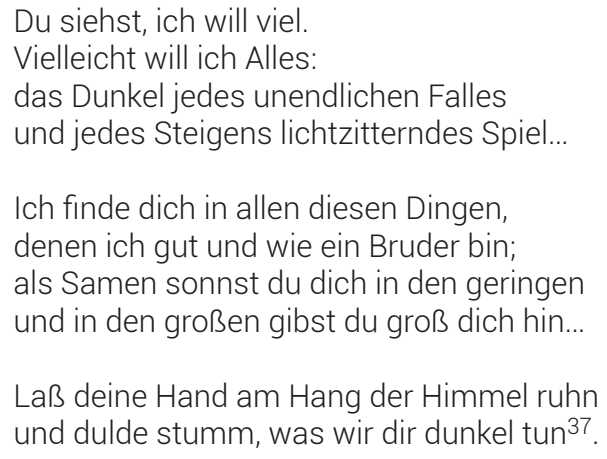

Ici les attributs de la première personne désignent une activité de la deuxième. Les images ne désignent pas des objets en tant que tels mais une activité sensible de la deuxième personne. Dans les deux derniers vers cités, il est remarquable que le «toi» semble être la condition de possibilité de l'activité de la personne parlante. Mais c'est dans la réunion ou l'unification des deux entités d'abord séparées que se désigne cette activité spécifique, un faire qui demeure obscur, indiscernable dunkel tun- et qui est aussi «te trouver» -ich finde dich in allen Dingen. La liberté affirmée à la fin n'est une liberté que parce qu'elle est autorisée; cette réussite de la liberté est aussi celle dans laquelle l'euphonie atteint un haut degré d'élaboration avec un emboîtement des effets sonores les uns dans les autres - und dulde, dulde stumm, was wir, wir dir, dunke tun. Le sens des poèmes est ainsi exemplifié par ce phénomène d'assonance, par leur réussite phonique. Et l'on sait qu'ils furent écrits par Rilke très rapidement et avec bonheur. Renvoyant à Dieu, le langage renvoie à sa pure sonorité de poésie. C'est là précisément ce que Paul Valéry notait à propos de la «parfaitement fidèle» traduction des Euvres spirituelles du mystique poète Jean

37. Rilke, Das Stunden Buch, Erstes Buch, Das Buch vom mönchischen Leben (1899), 1972 (1ère édition 1905). Rilke, Euvres 2, Poésie, pp. 93, 95-96 : «Tu vois, je veux beaucoup. / Peut-être tout: / I'obscurité des chutes infinies / et le jeu scintillant de toute remontée... Je te trouve en toutes ces choses / que j'aime fraternellement; / semence, tu te chauffes au soleil des petites, / et dans les grandes avec grandeur tu t'accomplis... Laisse ta main aux flancs des cieux / et supporte en silence l'errance obscure de nos gestes» (traduction de Jacques Legrand). 
de la Croix par le P. Cyprien: «Ceci chante tout seul». Telle la voix entendue par saint Augustin dans le jardin de Milan.

C'est à Capri puis à Duino, entre Venise et Trieste, entre octobre 1911 et mai 1912, que Rainer Maria Rilke a vécu avec intensité une expérience double et successive, d'abord de forme mystique puis poétique, qui l'amène à reconnaître, du moins à entrevoir et pressentir, peut-être à appeler en l'exprimant, ce qu'il dénomme Weltinnenraum, l'espace intérieur du monde qui est aussi bien l'intimité des choses que celle des personnes en communication de force pure les unes avec les autres. Dans cette étendue en nous, explique-t-il à l'occasion de l'expérience de Capri, «l'infini pénétra de toutes parts avec une telle intimité qu'il put croire sentir dans sa poitrine le poids léger des étoiles qui venaient de s'allumer ${ }^{38}$. Si depuis des années Rilke se passionne pour la vie des saints et compose de nombreux poèmes sur ce thème, il écrit alors à Duino en quelques jours Das Marienleben et il commence la rédaction des Élégies. Un poème daté d'août 1914, destiné à définir la notion d'espace intérieur du monde, revient sur l'expérience spirituelle vécue par le poète à Duino:

Durch alle Wesen reicht der eine Raum:

Weltinnenraum. Die Vögel fliegen still

durch uns hindurch. Oh, die ich wachsen will,

ich seh hinaus, und in mir wächst der Baum ${ }^{39}$

Un arbre aurait ainsi ouvert au poète une origine mystérieuse de la poésie. Or, cet arbre, c'est un olivier qui, à la fin de 1911, dans un jardin du château de Duino, transmet à Rilke une vibration aussi merveilleuse qu'effrayante provenant d'une «source obscure». Surpris d'un étrange sentiment et d'un frisson, Rilke a alors l'impression d'«avoir traversé la nature pour en sortir de l'autre côté» ${ }^{40}$. Un peu plus tard, en janvier 2012, alors qu'il marche en surplombant la mer, il croit soudain entendre parmi les rugissements du vent et des vagues une voix lui dire la phrase suivante: Wer, wenn ich schriee, hörte mich denn aus der Engel Ordnungen? -Qui donc, si je criais, parmi les hiérarchies des anges, m'entendrait?- Ce sera la première phrase des Élégies. C'est ainsi que la poésie naît à partir d'une ouverture entre la terre et le ciel, provenant de la terre et articulée avec le ciel, médiatisée par un arbre et des vents. Cette ouverture est bien la destination même de la poésie.

Car ici, comme dans les poèmes de Jean de la Croix, c'est à travers tous les êtres que l'on atteint «l'espace intérieur du monde», «ampleur intime», selon Rilke, ce Dieu «plus intime que le plus intime de nous-même», selon la belle expression de saint Augustin. C'est alors que l'on voit dans ce «toi», dans l'autre, sa propre étendue et le monde. Mais Rilke repousse, comme on le sait, la solution chrétienne.

38. Rilke, Euvres 7, Prose, Fragments en prose, Aventure II, p. 299.

39. Cité par Blanchot, 1955, p. 174 : «A travers tous les êtres passe l'unique espace: / espace intérieur du monde. Silencieusement volent les oiseaux / tout à travers nous. 0 moi qui veux croître, / je regarde au-dehors et c'est en moi que l'arbre croît».

40. Rilke, Euvres 7, Prose, Fragments en prose, Aventure I, p. 297. 
13 Mi Amado las montañas,

Los valles solitarios nemorosos,

Las ínsulas extrañas,

Los ríos sonorosos,

El silbo de los aires amorosos,

14 La noche sosegada,

En par de los levantes de la aurora,

La música callada,

La soledad sonora,

La cena que recrea y enamora ${ }^{41}$.

Todo lo que aquí se declara está en Dios eminentemente en infinita

manera... el alma siente ser todas las cosas Dios en un simple ser... ${ }^{42}$

L'Aimé est bien ici l'espace intérieur du monde - pure poésie- parcouru par l'aimée; en lui se révèle le monde, comme le souligne la traduction du carme du XVIlème siècle. La nature entière se transforme: les fleuves, les arbres, les prairies, la lumière elle-même de la nuit ou du jour, sont dans la présence aimée:

J'ay en mon bien-aymé les monts

Et les vallées solitaires,

Les fleuves bruyans et profonds,

Avec les Isles estrangeres,

Le souffle des plus doux zephirs

Qui rafraichissent mes désirs.

La paisible et tranquille nuit,

Pareille à l'aube gracieuse:

La douce musique et sans bruit;

La solitude harmonieuse:

Le souper que donne l'amour,

Et recrée l'ame à son tour ${ }^{43}$.

Après avoir longuement et avec impatience attendu le voyage d'Espagne, Rilke entre enfin en Espagne le 31 octobre 1912 par Bayonne. II arrive à Tolède deux jours plus tard, un 2 novembre, jour des Morts. En recherche de l'accomplissement d'une initiation et d'une catharsis, il reste un mois à Tolède, «le voyage des voyages». Pour lui le paysage de Tolède, si bien décliné par Greco, se fait monde, création, montagne et abîme, genèse véritable. Tolède est la «montagne de la révélation» où l'invisible devient visible, Tolède confère un don de prophétie au poète. Le paysage intérieur du poète trouve en effet sa relation avec le paysage castillan en une communion mystique de type cosmique et existentiel. L'Espagne de Rilke est alors une Espagne baroque où Velázquez et surtout Greco occupent une place privilégiée à côté des mystiques et du théâtre du Siècle d'Or, une Espagne configurée par le

41. San Juan de la Cruz, Cántico espiritual y poesía completa, pp. 19-20.

42. San Juan de la Cruz, Cántico espiritual y poesía completa, p. 93.

43. Jean de la Croix, Les Cantiques spirituels, Cantiques entre l'ame et Jesus-Christ son espoux, p. 52. 
romantisme allemand. Au début du mois de décembre, ayant quitté Tolède, Rilke visite Cordoue et Séville, puis il arrive à Ronda le 7 décembre et y demeure plus de deux mois avant de se rendre à Madrid dans l'intention de contempler les œuvres de Goya et Greco. Puis il quitte Madrid pour Paris à la fin du mois de février $1913^{44}$.

Citons ici le poème envoyé à Lou Andreas-Salomé en juin 1914, intitulé Wendung parce que Rilke a la conviction d'avoir accompli depuis le voyage en Espagne le «virage décisif», le «tournant» ou la conversion qu'il désirait. Rilke choisit de poser en exergue de son poème la phrase de son ami intime, le philosophe Rudolf Kassner: Der Weg von der Innigkeit zur Größe geht durch das Opfer - Le chemin qui mène de l'intimité à la grandeur passe par le sacrifice. Tout au long du poème, visions, parfums, silence en représentations sensibles très fortes se joignent, comme autant d'étapes d'un parcours, évoquant le paysage cosmique et existentiel de l'Espagne. L'espace parcouru serait ici à la fois intimité et dehors, déjà intimité spirituelle et réalité du dehors. Arrivé à l'extrême limite du parcours de la contemplation, le poète comprend que c'est l'œuvre du cœur qui importe, le cœur devenu œuvre poétique, chant, pure poésie, énergie vitale. La limite qui est aussi la mort nous repousse vers ce que nous sommes et nos propres représentations, nous détourne du tout autre. Accéder à l'autre côté, être capable de tournant, ce serait entrer dans la liberté de ce qui est libre de limite quand l'être voit dans ce tout autre, dans l'aimé, sa propre étendue. Aimer, c'est avoir quelqu'un devant soi; c'est être l'aimée à la poursuite de l'aimé, ne regarder que lui et jamais au-delà de lui.

Denn des Anschauns, siehe, ist eine Grenze.

Und die geschaute Welt

will in der Liebe gedeihn.

Werk des Gesichts ist getan,

tue nun Herz-Werk

an den Bildern in dir, jenen gefangenen; denn du

überwältigtest sie: aber nun kennst du sie nicht ${ }^{45}$.

Buscando mis amores,

Iré por esos montes y riberas,

Ni cogeré las flores,

Ni temeré las fieras,

Y pasaré los fuertes y fronteras,

chante l'épouse du Cántico espiritual en œuvre d'amour à la poursuite de son aimé.

44. Le livre de Jaime Ferreiro Alemparte, España en Rilke (1966), reste l'ouvrage de référence.

45. Rainer Maria Rilke- Lou Andreas-Salomé, Lettre du 20 juin 1974, éd. Ernst Pfeiffer, 1952. Traduction française de Pierre Klossowski, Correspondance Rainer Maria Rilke et Lou Andreas-Salomé, 1967: «Tournant - Voici il est posé une limite au regard. / Et l'univers regardé / veut s'épanouir dans l'amour. L'œuvre de la vision est faite, / fais désormais l'œuvre du cœur / auprès des images en toi, ces images captives; car tu / les avais dominées : mais tu restes sans les connaître». 
Et la traduction du P. Cyprien de la Nativité de la Vierge donne:

\author{
Cherchant les amours de mon cœur \\ J'iray par ces monts et rivages, \\ Sans y cueillir pas une fleur \\ Ny craindre les bestes sauvages: \\ Murs et remparts je forceray \\ Et les frontières passeray.
}

Ainsi trouve son accomplissement, pour reprendre l'intuition de Rilke si bien précédée par le P. Cyprien de la Nativité de la Vierge, cette liberté dégagée de toute représentation sensible et de tout enfermement et de toute limite pour s'abandonner à la seule œuvre d'amour. La perspective synesthésique du mystique Jean de la Croix atteint ici sa finalité. II est remarquable que la traduction du P. Cyprien désigne clairement ce passer au-delà, de l'autre côté: l'entrée par l'amour et par le poème dans la disparition et un autre commencement, le «nulle part sans néant» de la Huitième Élégie de Rilke.

Maria Zambrano remarque à propos du Cantique spirituel:

Tel est, selon nous, le fondement de toute mystique: l'amour qui naît dans la chair (tout amour «premier» est charnel), doit pour se réaliser, se détacher de la vie, doit aussi se convertir comme, selon Platon, devait le faire la connaissance ${ }^{46}$.

Installé à Muzot à partir de juillet 1921, Rilke découvre dans le paysage du Valais ce paysage «de présence» qui lui permet d'évoquer et reconstruire le paysage «d'absence». Ce paysage absent, invisible est le paysage de l'Espagne, le dernier paysage qu'il ait vécu intensément, comme il l'affirmera à maintes reprises. Le paysage valaisan joue ici un rôle médiateur, offrant ses ressemblances pour une ultime révélation intérieure. Rilke y complète les Élégies en quelques jours en février 1922. La Huitième Elégie, dédiée à Rudolf Kassner, est une méditation qui reprend et souligne une conviction majeure de Rilke: ce qui entrave l'être humain dans son mouvement vers «l'ouverture profonde», «ce pur espace où s'ouvrent sans fin les fleurs», «ce 'nulle part' sans néant: la pureté que rien ne surveille», c'est l'angoisse de la mort. Car le monde ouvert est celui que voient les anges et, avec eux, ceux qui ne séparent pas la vie de la mort.

\author{
Mit allen Augen sieht die Kreatur \\ das Offene. Nur unsre Augen sind \\ wie umgekehrt und ganz um sie gestellt \\ als Fallen, rings um ihren freien Ausgang. \\ Was draußen ist, wir wissens aus des Tiers \\ Antlitz allein; denn schon das frühe Kind \\ wenden wir um und zwingens, daß es rückwärts \\ Gestaltung sehe, nicht das Offne, das \\ im Tiergesicht so tief ist. Frei von Tod.
}


Ihn sehen wir allein; das freie Tier

hat seinen Untergang stets hinter sich

und vor sich Gott, und wenn es geht, so gehts

in Ewigkeit, so wie die Brunnen gehen.

Wir haben nie, nicht einen einzigen Tag,

den reinen Raum vor uns, in den die Blumen

unendlich aufgehn. Immer ist es Welt

und niemals Nirgends ohne Nicht: das Reine,

Unüberwachte, das man atmet und

unendlich weiß und nicht begehrt... ${ }^{47}$

La créature, c'est-à-dire l'être animal qui vit dans son innocence originelle, voit l'«Ouvert» de ses yeux; elle est un regard tourné vers lui, qui avance librement vers son but. Les yeux de l'être humain le maintiennent par la considération effrayante et angoissante de sa mort propre dans la limite de l'espace et du temps; ils le détournent de l' "Ouvert». Et ils l'excluent aussi par l'avidité de la possession des choses de l'accès véritable au réel des choses. L'animal, pour sa part, peut entrer dans la réalité sans avoir à en être le centre, car son être est pour lui sans fin, puisqu'il ne voit pas sa mort et n'en a pas d'angoisse; il ouvre son regard sur la chose, étant ainsi dans la chose même et non dans sa représentation, selon un «pur rapport»; il ne voit pas son anéantissement, mais Dieu qui lui est ouvert, l'éternité dans laquelle il entre. Une des dimensions thématiques centrales de l'œuvre de Rilke est cette mise en demeure visant à transformer radicalement notre manière d'être dans le monde: parce que nous ne savons jamais reconnaître l'espace pur, affranchi, parce que nous n'admettons pas la mort comme le complément nécessaire de la vie, nous avons exclu et nous nous sommes exclus de l'infini. II nous faut nous retourner, regarder librement au-delà des limites du monde, cesser de prendre congé. Si nous reconnaissons que la mort est le côté de l'existence qui n'est pas tourné vers nous, le monde peut alors nous apparaître dans sa totalité.

N'est-ce pas là le principe des Cantiques spirituels de Jean de la Croix? L'«animal libre» «qui a son déclin derrière lui / et devant lui Dieu» et «quand il avance, c'est dans l'éternité, comme coulent les sources», n'est-il pas l'aimée qui parcourt le monde à la recherche de son bien aimé?

Les deux dernières Élégies, désignant par l'union de la vie et de la mort la recréation d'un monde total enfin sauvé, annoncent la possible advenue du poète dans

47. Rilke, Les Élégies de Duino - Duineser Elegien, pp. 82-83. Traduction française: «De tous ses yeux la créature voit / "I'Ouvert". Nos yeux seuls sont / comme inversés et tout à fait placés autour d'elle / tels des pièges pour encercler sa libre issue. / Ce qui est au-dehors, nous ne le connaissons / que par les yeux / de l'animal. Car le jeune enfant déjà / nous le retournons et le contraignons à regarder en arrière, / le monde des formes, non pas l'Ouvert, qui / dans le visage de l'animal est si profond. Libre de mort. / Elle, nous ne voyons qu'elle; l'animal libre / a toujours son déclin derrière lui / et devant lui Dieu, et lorsqu'il avance, il avance / dans l'Eternité, comme coulent les sources. / Mais nous, jamais, pas un seul jour, nous n'avons / devant nous le pur espace, dans lequel les fleurs / infiniment s'épanouissent. Toujours c'est le monde, / et jamais ce qui n'est nulle part et que rien ne limite: / le pur, l'insurveillé, que l'on respire, / que l'on sait infini et ne convoite pas ». 
I'«Ouvert» qu'il vient de percevoir. Dès 1914, Rilke avait écrit: Werk des Gesichts ist getan, / tue nun Herz-Werk - L'œuvre de l'œil est accomplie, / fais maintenant celle du cœur... La neuvième Élégie rappelle que toutes les choses passent et se transforment, que nous voulons désormais passer, que nous voulons la transformation. D'où les injonctions du poète: Wollen es werden - Vouloir le devenir ${ }^{48}$, Also die Schmerzen - Donc, les souffrances ${ }^{49}$. Le chemin de la vraie vie, chemin de salvation, est celui de la souffrance, nécessaire pour atteindre le réel. La souffrance et la mort, seules, peuvent permettre d'ajouter l'infini au fini. Erde, ist es nicht, was du willst... Was, wenn Verwandlung nicht, ist dein drängender Auftrag? - Terre, n'est-ce pas ce que tu veux... Quelle mission imposes-tu, si ce n'est la transformation? ${ }^{50}$ est-il encore recommandé dans la neuvième Élégie. Après ce consentement à la terre, dans la dixième Élégie c'est l'au-delà qui s'ouvre et s'offre au cœur de I'homme plus qu'à sa raison. Si la terre, chaque année, se referme sur elle-même, comme une femme sur son fruit, c'est pour revivre au printemps, grâce à la pluie.

Maurice Blanchot a montré dans son ouvrage L'espace littéraire, qui est un essai d'élucidation du fait que «quelque chose comme l'art ou la littérature existe», que l'œuvre de Rilke s'inscrit dans la perspective de l'expérience de Mallarmé, ce maître de Valéry. En effet, ce dernier «en creusant le vers», selon sa propre expression, est entré dans le temps de la détresse qui est celui de l'absence de Dieu -le néant ou la nada de Jean de la Croix - et il rencontre sa mort comme abîme ${ }^{51}$. De même Rilke fait de la poésie un rapport avec l'absence. Mais, à la différence de Mallarmé pour qui l'absence reste la force du négatif, ce qui nous délivre de la pesanteur des choses, pour Rilke l'absence est aussi la présence des choses, l'intimité de l'être où se rassemble le désir de tomber vers le centre comme une suprême possibilité, un mouvement, l'approche de la grâce, de l'ouverture poétique. II ne s'agit pas pour Rilke de surgir en Dieu mais d'être fidèle à la terre, à la plénitude et à la surabondance joyeuse grâce à la parole poétique et à la pure poésie qui métamorphose le visible en invisible.

Où l'art prendrait-il son point de départ si ce n'était dans cette joie et dans cette tension d'un commencement infini?52, écrit Rilke le 23 février 1921.

Dans le Cantique spirituel de Jean de la Croix, c'est bien la terre en sa dense épaisseur qui accueille la joie de l'expérience mystique et de la métamorphose du visible en invisible, de la beauté de l'aimé en celle de l'aimée:

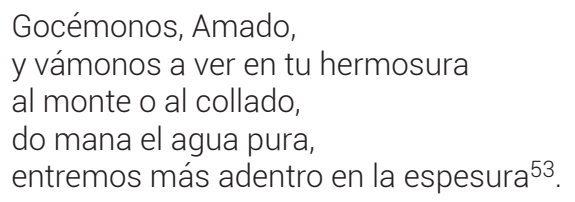

48. Rilke, Les Élégies de Duino - Duineser Elegien, pp. 88-89.

49. Rilke, Les Élégies de Duino - Duineser Elegien, pp. 90-91.

50. Rilke, Les Élégies de Duino - Duineser Elegien, pp. 92-93.

51. Blanchot, 1955, pp. 37-38.

52. Rilke, Euvres 3, Correspondance : Lundi 23 février 1921, A Merline (Baladine Klossowska), p. 461.

53. San Juan de la Cruz, Cántico espiritual y poesía completa, p. 41. 
... lo cual será transformándome a mí en tu hermosura, y así te veré yo a ti en tu hermosura, y tú a mí en tu hermosura, y tú te verás en mí en tu hermosura, y yo me veré en ti en tu hermosura; y parezca yo tú en tu hermosura... y mi hermosura sea tu hermosura y tu hermosura mi hermosura... 54

A la fin du Cantique spirituel, toutes les expériences sensibles s'abolissent désormais en un heureux mouvement descendant, car «les puissances et les sens du corps» entrent dans le seul recueillement intérieur. Commentant les vers «Y la caballería / a vista de las aguas descendía», Jean de la Croix précise:

El alma esposa por «la caballería» entiende las potencias de la parte sensitiva, así interiores como exteriores, las cuales dice la esposa que en este estado descienden a vista de estas aguas espirituales... ella con sus potencias sensitivas y fuerzas naturales se recoge a participar y gozar en su manera de las grandezas espirituales que Dios está comunicando al espíritu... y dice aquí el alma que «descendía» y no otro vocablo alguno, para dar a entender que todas estas potencias descienden y bajan de sus operaciones naturales, cesando de ellas, al recogimiento interior... 55

Les Élégies de Duino, chants de mort, s'achèvent sur une image de bonheur, celle de «la pluie qui tombe sur le sombre royaume de la terre au printemps». Rilke commente ainsi ce mouvement de descente, de chute:

Und wir, die an steigendes Glück

Denken, empfänden die Rührung,

die uns beinah bestürzt,

wenn ein Glückliches fällt56

En 1923, à Muzot, Rilke qui achève alors la traduction de Charmes de Paul Valéry écrit le 23 avril à son épouse Clara: «Ces dernières semaines, ma plume a été toute occupée à recopier les assez nombreuses traductions que m'a values cet hiver -d'admirables poèmes de Paul Valéry - et jamais, me semble-t-il, je n'ai été un traducteur plus exact et plus heureux» ${ }^{57}$. Rilke fera la connaissance de Valéry le 5 avril 1924: «Cher Rilke, qui me paraissiez enfermé dans un temps trop pur...», écrira Valéry par la suite ${ }^{58}$. Etre enfermé dans un temps trop pur, c'est aussi le détachement de la vie.

Paul Valéry et Rainer Maria Rilke ont-ils évoqué les Eruvres spirituelles de Jean de la Croix lors de leur rencontre?

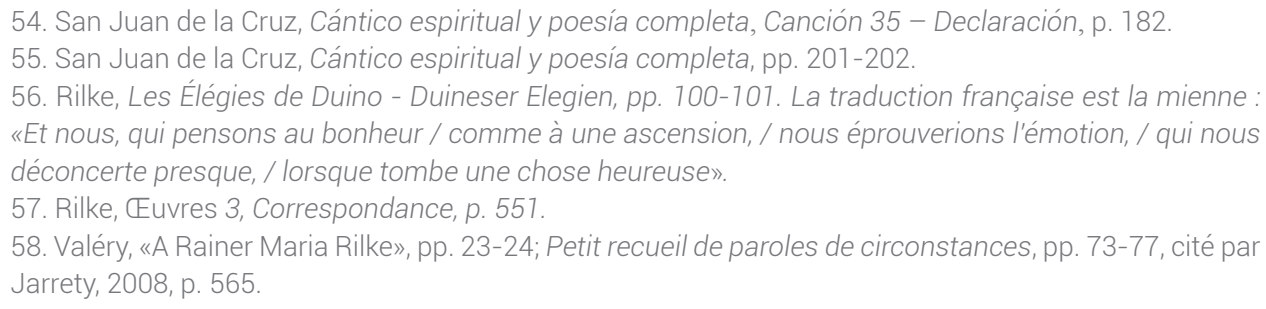


Ce qui est certain c'est qu'après cette rencontre, à partir de 1924, dans les dernières années de sa vie, Rainer Maria Rilke découvre et lit dans le texte original la Noche oscura del alma de Jean de la Croix ${ }^{59}$. On ne sait pas si Rilke a laissé un témoignage des impressions de sa lecture. Mais il est certain qu'il ne pouvait que constater ses affinités avec le mystique castillan. II décide alors de réélaborer et achever le cycle des Poèmes à la nuit, commencé au cours de son voyage en Espagne. Entre août et octobre 1924 sont composés quatre poèmes à la nuit, en lesquels sont bien visibles les résonnances du paysage à la fois cosmique et existentiel de l'Espagne ${ }^{60}$. Le thème de la nuit trouve ici peut-être sa phase ultime de pureté et dépouillement poétique en ouverture et liberté.

Nachthimmel und Sternenfall.

Der Himmel, gro $\beta$, voll herrlicher Verhaltung, ein Vorrat Raum, ein Überma $\beta$ von Welt ${ }^{61}$.

Et encore:

Dort mündete, nach Sturz und Widerstand des Laufes, das Eröffnete genießend, in stillen Armen auseinanderfließend, der breitgewordene, der Adorant ${ }^{62}$.

An das Stillende hinaufgekehrt, hab ich mich zur heilen Nacht entschlossen, meine Sinne sind mir abgeflossen und das Herz ist namenlos vermehrt ${ }^{63}$.

Jean de la Croix écrivait dans la Noche oscura:

¡Oh noche que guiaste!,

¡oh noche amable más que la alborada!

¡oh noche que juntaste

Amado con amada,

amada en el Amado transformada!

... con su mano serena...

Y todos mis sentidos suspendía..

Quedéme y olvidéme,

el rostro recliné sobre el Amado;

59. Cf. Ferreiro Alemparte, 1966, pp. 315-317.

60. Rilke, Poèmes à la nuit. Die Gedichte, 1906 bis 1926.

61. Rilke, Poèmes à la nuit, p. 87. Traduction française: «Ciel nocturne et chute d'étoiles. / Le ciel, grand, plein de retenue splendide, / une provision d'espace, un excès de monde».

62. Rilke, Poèmes à la nuit, p. 59. Traduction française: «Là-bas se jetait, après la chute et la résistance / de sa course, jouissant de l'Ouvert, / dans le cours divergent de bras paisibles, / celui qui est devenu ample, l'Adorant».

63. Rilke, Poèmes à la nuit, p. 61. Traduction française: «Tourné attentivement vers ce qui apaise, / je me suis résolu à la nuit intacte, / mes sens se sont écoulés de moi / et le cœur indiciblement en est multiplié». 
cesó todo y dejéme,

dejando mi cuidado

entre las azucenas olvidado ${ }^{64}$

La traduction du P. Cyprien présentée par Paul Valéry est:

O Nuict qui me conduis à point!

Nuict plus aymable que l'aurore!

Nuict heureuse qui as conjoint

L'aymée à l'Aymé, mais encore

Celle que l'amour a formé,

Et en son Amant transformé! 65

...sa douceur tint en suspens

L'entier usage de mes sens.

Je me tins coy, et m'oubliai,

Penchant sur mon amy ma face,

Tout cessa, je m'abandonnay,

Remettant mes soins à sa grâce:

Comme estans tous ensevelis

Dans le beau parterre des Lys ${ }^{66}$.

Citons encore Rainer Maria Rilke:

Nun erst, Nachtstunde, bin ich ohne Angst

Und darf in aufgeblühtem Schauen stehen

Da du für dein unendliches Geschehen

Mein ungenügendes Gesicht verlangst.

Jetzt tritt aus ihm die Ähnlichkeit hervor. ${ }^{67}$

O von Gesicht zu Gesicht

Welche Erhebung ${ }^{68}$.

Le poème est l'espace où tout retourne à l'être profond, où tout s'abandonne et meurt, mais où l'abandon accompagne la transformation et la vie nouvelle. L'aimée, adoratrice -adorante- de son aimé, visage contre visage, disparaît dans l'ampleur intime, parfumée et belle, enveloppante et silencieuse du monde et du poème, afin de s'apprêter à surgir en son aimé, en Dieu.

Pues ya si en el ejido

De hoy más no fuere vista ni hallada,

64. San Juan de la Cruz, Cántico espiritual y poesía completa, pp. 206-207.

65. Jean de la Croix, Les Cantiques spirituels, p. 38.

66. Jean de la Croix, Les Cantiques spirituels, p. 40.

67. Rilke, Poèmes à la nuit, pp. 62-63. Traduction française: «Ce n'est qu'à présent, heure nocturne, que je suis sans crainte / et qu'il m'est permis de me tenir là, les yeux levés, épanouis, / puisque pour l'advenue de ton infinitude tu / demandes mon insuffisant visage. / Maintenant voici que la ressemblance s'en dégage». 68. Rilke, Poèmes épars (1907-1926), pp. 62-63. Traduction française: «Oh, du visage au visage / quelle élévation». 
Diréis que me he perdido,

Que, andando enamorada,

Me hice perdidiza y fui ganada 69

Ce que traduit ainsi le P. Cyprien:

Que si desormais en ces prez,

L'on ne me trouve et n'y suis veuë:

Et si l'on s'enquiert vous direz

Que vraiment je me suis perduë,

Qu'esprise d'un amour ardent,

Je me gaignay en me perdant ${ }^{70}$.

Ainsi il y a la parole qui définit et celle qui ne trace pas de chemin, qui semble égarée mais qui est l'ouverture totale de la vie, «la parole irrationnelle de la poésie... lancée dans l'aventure de dire l'indicible... à partir de l'immense territoire qu'en son errance elle parcourt », écrit Maria Zambrano ${ }^{71}$. Le sens de l'énoncé et le mode du dire se recouvrent dans la poésie. Pensée et poésie sont ici unies, comme l'a si exemplairement compris le P. Cyprien de la Nativité de la Vierge, traducteur de Jean de la Croix et de son expérience mystique. A sa suite, Valéry et Rilke se rejoignent dans leur conviction que le langage poétique est catégorie constitutive du sens et c'est bien ce qu'ils s'attachent à mettre en œuvre dans leur propre pratique de poésie et de traduction. Après eux, le traducteur des Élégies de Duino, Joseph-François Angelloz, dont nous avons retenu la traduction, s'est ainsi expliqué:

La grandeur (des Élégies), qui joint à la beauté plastique l'harmonie du chant, est d'une telle densité qu'elles inspirent presque au traducteur le même effroi que les anges... Traduire les Élégies... paraît un défi; ne sont-elles pas elles-mêmes un défi à tout ce qu'on appelait communément «poésie»? Elles révèlent, en particulier, plus qu'aucun autre poème allemand, un combat avec la langue, dont Rilke ne sortit vainqueur qu'en reculant à l'extrême les possibilités qu'elle offre. Mon effort constant fut de transposer son œuvre aussi fidèlement que possible, avec l'ardent désir de n'être qu'un médiateur spirituel, qui voudrait se faire oublier du lecteur pour lui permettre de mieux comprendre et aimer l'œuvre rilkéenne.

1926, I'année de la mort de Rainer Maria Rilke, est aussi celle de la célébration du bicentenaire de la canonisation de Jean de la Croix et de sa proclamation comme Doctor Ecclesiae. L'intérêt et l'admiration pour l'œuvre du saint ne cessent désormais plus de croître, non seulement parmi les philosophes et les théologiens, mais aussi parmi les écrivains et les artistes. En Espagne, pour le groupe de poètes dit de la generación del 27, qui apparaît entre 1923 et 1927, Jean de la Croix devient un modèle de modernité poétique, alliant rupture et perfection esthétique. Se réclamant de la «poésie pure» des «symbolistes français» Mallarmé et Valéry, Federico García Lorca (1898-1936) compose dans les dernières années de sa vie des So-

69. San Juan de la Cruz, Cántico espiritual y poesía completa, p. 26.

70. Jean de la Croix, Les Cantiques spirituels, p. 56.

71. Zambrano, 2003, pp. 156-159. 
netos de amor oscuro, dans lesquels il adopte souvent le langage amoureux de la mystique de Jean de la Croix; Dámaso Alonso s'inspire de l'œuvre johannique dans Oscura noticia, Hijos de la ira (1944) et Hombre y Dios (1955).

C'est ainsi que les harmoniques de l'expérience spirituelle, mystique de Jean de la Croix au XVlème siècle en Espagne, se déploient en persistances et reprises poétiques infinies, toujours nouvelles, toujours recommencées. A partir de ses poèmes majeurs se dessinent des entrelacements inattendus, radicaux. Ainsi s'entrelacent et se croisent la traduction effectuée au XVIIème siècle par le carme P. Cyprien, la découverte de cette traduction par le poète Paul Valéry au début du XXème siècle, en parfaite conjonction avec sa propre recherche, ou encore leur résonnance dans l'œuvre poétique de Rainer Maria Rilke, amoureux des «paysages» de l'Espagne, lecteur de Paul Valéry et de Jean de la Croix. Tout cela en attente d'autres entrelacements et d'autres croisements. C'est toute une synesthésie multiple, associée aux expériences sensibles, physiques ou spirituelles, de la vie, qui s'éprouve ainsi, pour se déployer en poésie pure par-delà les différences de langues, d'espaces et de temps.

... O reine Uebersteigung!... Und alles schwieg. Doch selbst in der Verschweigung, ging neuer Anfang, Wink und Wandlung vor ${ }^{72}$.

En par de los levantes de la aurora. Este sosiego y quietud no es... sino sosiego y quietud en luz divina en conocimiento de Dios nuevo, en que el espíritu está suavísimamente quieto, levantado a luz divina ${ }^{73}$.

\section{BIBLIOGRAPHIE}

Blanchot, Maurice, L'espace littéraire, Paris, Gallimard, 1955.

Böschenstein, Bernhard, «Rainer Maria Rilke, poète français, à l'écoute de Paul Valéry», Études germaniques, 262, 2011-2012, pp. 289-296.

Courcelles, Dominique de, Le «Dialogue» de Catherine de Sienne, Paris, Cerf, 1999.

Courcelles, Dominique de, Augustin génie de l'Europe, Paris, Lattès, 1998. Trad. española: Agustín o el genio de Europa, Santiago de Chile, Dolmen, 1998.

Courcelles, Dominique de, «María Zambrano y el lenguaje De la aurora», en Mística y creación en el siglo XX, Barcelona, Herder, 2006, pp. 57-80.

Ferreiro Alemparte, Jaime, España en Rilke, Madrid, Taurus, 1966.

Jarrety, Michel, Valéry, Paris, Fayard, 2008.

72. Rilke, Les Élégies de Duino - Duineser Elegien, pp. 150-151. Traduction française: «... O pure élévation! / ... Et tout se tut. Pourtant, au sein même de l'unanime silence / s'accomplit un nouveau recommencement, signe et métamorphose».

73. San Juan de la Cruz, Cántico espiritual y poesía completa, p. 103. 
Jean de la Croix, Les Cantiques spirituels traduits en vers français par le R. P. Cyprien, texte présenté par Paul Valéry, Paris, Desclée de Brouwer, Les Carnets, 1996.

Juan de la Cruz, san, Cántico espiritual y poesía completa, ed., prólogo y notas Paola Elia y María Jesús Mancho con un estudio preliminar de Domingo Ynduráin, Barcelona, Crítica, 2002.

Juan de la Cruz, san, Noche oscura, en Obras completas, Madrid, Biblioteca de Autores Cristianos, 1989.

Juan de la Cruz, san, Subida del Monte Carmelo, en Obras completas, Madrid, Biblioteca de Autores Cristianos, 1989.

Lara Garrido, José, «La primacía de la palabra como música y memoria en San Juan de la Cruz», en Hermenéutica y mística: San Juan de la Cruz, ed. José Ángel Valente y José Lara Garrido, Madrid, Tecnos, 1995.

López-Baralt, Luce, Prologue, en Juan de la Cruz, Obra completa, 1, Madrid, Alianza Editorial, 1991.

Morier, Henri, «Harmoniques», en Dictionnaire de poétique et de rhétorique, Paris, PUF, 1981, pp. 499-500.

Picard, Michel-Jean, «Cyprien de la Nativité de la Vierge», Dictionnaire de spiritualité ascétique et mystique, vol. 2, Paris, Beauchesne, 1933, pp. 2669-2671.

Rilke, Rainer Maria, Euvres 1, Prose, édition établie et présentée par Paul de Man, Paris, Seuil, 1972.

Rilke, Rainer Maria, Euvres 2, Poésie, édition établie et présentée par Paul de Man, Paris, Seuil, 1972.

Rilke, Rainer Maria, Poèmes épars (1907-1926), choisis, traduits et présentés par Philippe Jaccottet, édition bilingue, Paris, Seuil, 1972.

Rilke, Rainer Maria, Les Élégies de Duino - Duineser Elegien. Les Sonnets à Orphée - Die Sonette an Orpheus, trad. et préface, Joseph-François Angelloz, Paris, Garnier-Flammarion, 1992.

Rilke, Rainer Maria, Poèmes à la nuit, trad. et présentation Gabrielle Althen et JeanYves Masson, Lagrasse, Verdier, 1994.

Rilke, Rainer Maria, Die Gedichte, 1906 bis 1926, Frankfurt-am-Main, Insel Verlag, 1986.

Rilke, Rainer Maria, Das Stunden Buch, Erstes Buch, Das Buch vom mönchischen Leben (1899), Frankfurt-am-Main, Insel Verlag, 1972 (1ère éd. 1905).

Rilke, Rainer Maria, Euvres 3, Correspondance, éd. Philippe Jaccottet, trad. Blaise Briod, Philippe Jaccottet et Pierre Klossowski, Paris, Seuil, 1976.

Rilke, Rainer Maria, Lettre du 20 juin 1914, Correspondance Rainer Maria Rilke-Lou 
Andreas-Salomé, éd. Ernst Pfeiffer, Zürich, Max Niehans Verlag - Wiesbaden, Insel Verlag, 1952.

Rilke, Rainer Maria, Correspondance Rainer Maria Rilke et Lou Andreas-Salomé, préface et trad. de Pierre Klossowski, Paris, Le Nouveau Commerce-Cahier 9, printemps-été 1967.

Valéry, Paul, «A Rainer Maria Rilke», dans Reconnaissance à Rilke, Cahiers du mois, août 1926, pp. 23-24.

Valéry, Paul, Petit recueil de paroles de circonstances, Le Plaisir du Bibliophile, 1926.

Valéry, Paul, Cahiers, Paris, Rouart, 1941.

Zambrano, Maria, Philosophie et poésie, trad. Jacques Ancet, Paris, José Corti, 2003. 